Portland State University

PDXScholar

3-19-2021

\title{
Self-Perception of Stuttering Frequency Across the Lifespan: A Pilot Study
}

Natalia C. Newton

Portland State University

Follow this and additional works at: https://pdxscholar.library.pdx.edu/honorstheses

Part of the Speech and Hearing Science Commons, and the Speech Pathology and Audiology Commons

Let us know how access to this document benefits you.

\section{Recommended Citation}

Newton, Natalia C., "Self-Perception of Stuttering Frequency Across the Lifespan: A Pilot Study" (2021). University Honors Theses. Paper 974.

https://doi.org/10.15760/honors.998

This Thesis is brought to you for free and open access. It has been accepted for inclusion in University Honors Theses by an authorized administrator of PDXScholar. Please contact us if we can make this document more accessible: pdxscholar@pdx.edu. 
Self-Perception of Stuttering Frequency Across the Lifespan: A Pilot Study

\author{
Natalia Newton \\ An Undergraduate Honors Thesis Submitted in Partial Fulfillment of the \\ Requirements for the Degree of \\ Bachelor of Science \\ in \\ University Honors and Speech and Hearing Sciences \\ Thesis Advisor \\ Dr. Megann McGill, CCC-SLP \\ Portland State University \\ February 2021
}




\begin{abstract}
Purpose: The purpose of the current pilot study is to analyze the accuracy of self-rating of stuttering frequency as compared to clinician calculations of stuttering frequency in order to further examine possible factors that affect the accuracy of a stutterer's self-rating of their overt stuttering.

Method: Three participants (i.e., one child, one teen, one adult) self-rated their perceptions of their stuttering frequency on a scale of 1-5 after three telepractice speech therapy sessions. Each of the telepractice therapy sessions were recorded and reviewed asynchronously after the session to conduct a disfluency count with a 300-word sample and calculate the percentage of words stuttered for each client. The participants self-ratings were then analyzed for accuracy in comparison to the calculated percent of words stuttered.

Results: Pilot results revealed a potential inverse relationship between age and accuracy of clients' self-perception of stuttering frequency. The child client was the most accurate in their self-ratings and the adult client was the least accurate in their self-ratings of stuttering frequency. A consistent self-rating pattern was noted across the three therapy sessions, with two of the three clients reporting the same self-rating for all sessions, despite variability in clinician calculations of percent words stuttered.
\end{abstract}

Conclusion: Results of the current pilot study indicate that children may be more accurate in self-appraising their stuttering frequency when compared to teen and adult clients. The impact of covert stuttering on clients' self-ratings and clinical implications are discussed. 


\section{Introduction}

Stuttering is a disruption to the forward flow of speech. Stuttered speech may present aurally as single sound repetitions, audible sound prolongations, inaudible sound prolongations, and whole word repetitions (Harvard Health Publishing, 2018). Alongside these speech behaviors a variety of concomitant non-speech behaviors may occur. Some concomitant behaviors related to stuttering are tightening of the muscles in the face and neck, closing of the eyes or eye blinking, and tapping of the fingers or feet (Harvard Health Publishing, 2018). In addition to these overt behaviors of stuttering (e.g., repetitions, prolongations, blocks, secondary behaviors), people who stutter may also experience covert behaviors related to their stuttering. Covert behaviors may include escape strategies, such as "using verbal starters (e.g., "um...), creating distracting noises (e.g., clicking the tongue), abandoning the stuttered utterance, or physical responses (e.g., blinking eyes, moving the head, writhing the hands)" (Douglass et al., 2018, p. 1235).

Stuttering is only one category of disfluencies found in speech production. There are other disfluencies that are considered 'typical.' Typical disfluencies are also disruptions to the forward flow of speech, but are different from stuttering behaviors in that all speakers, including speakers who stutter, present these in conversational speech. These 'typical' disfluency behaviors are phrase repetitions (without any visible tension), interjections, and revisions of words after beginning to vocalize (ASHA, 2021).

\subsection{Stuttering Self-Perceptions}

Tichenor and Yaruss (2019) researched stuttering “as defined by adults who stutter" (p. 4356). This research showed that there were a variety of ways in which people experienced stuttering. The researchers received and analyzed 1,143 statements about how each individual who stutters would define stuttering. Those 1,143 statements could be placed under six overarching categories. These categories were: "loss of control/sensation of being stuck; affective/emotional reactions; behavioral reactions; 
cognitive reactions; limitations and impact; and perceived influence of listeners and conversation partners" (Tichenor \& Yariss, 2019, p. 4360). Although experiences of stuttering can be generalized in these overarching categories, each individual who stutters experiences variation in which category seems most prevalent in their life, and best explains their stuttering experience. The variations in those experiences can lead to differences in the way that one may perceive their stutter, and potentially alter their perceived stuttering frequency.

Variations in stuttering behaviors may also impact a stutterer's self-perception of stuttering frequency (O'Brian et al., 2004). O'Brian et al. (2004) reported that when many stuttering moments occurred very rapidly, clients rated their stuttering as being less severe. Conversely when there were a smaller number of prolonged stuttering moments, clients rated their stuttering higher on the severity scale (O’Brian et al., 2004). According to O'Brian et al. (2004), the manner in which a person experiences their own stuttering may impact their perception of the severity of their overt stuttering behaviors.

In addition, Alameer et al. (2017) reported that clients who stutter experienced "decreased fluency in real-life communication in comparison with clinical sessions" (p. 43). This phenomenon of increased fluency during therapy and decreased fluency outside of clinical environments is well documented in literature describing the variability of stuttering and the impact of communication situations on fluency. That is, when the stutterer experiences a social communication situation, they may perceive that their frequency of stuttering is increased.

\subsubsection{Covert Stuttering}

Another aspect of stuttering that may impact perceptions of frequency is covert stuttering. Covert stuttering can be defined as "the disproportionate use of secondary behaviors to hide a stutter" (Douglass et al., 2018, p. 1235). Covert behaviors can be used by individuals who stutter to be perceived as, or 'pass' as, someone who does not stutter. Covert stuttering engages all of the same cognitive and emotional effects of stuttering without the physical, overt stuttering behavior(s) (Douglass et al., 2018). Since an individual may experience the internal effects of stuttering without overt symptoms, it is possible that they will perceive their stutter as more severe than listeners/observers may report. 


\subsubsection{Age and Stuttering Perception}

Knodrashov and Tetnowski (2019) reported that younger children have a higher rating of negative self-perception of stuttering as compared to adults. Twenty-three participants ranging in age from 9-42 years old completed the Wright \& Ayre Stuttering Self-Rating Profile (WASSP) to report their attitudes towards their stuttering. Child and adult responses were compared and Knodrashov and Tetnowski (2019) found that, according to responses from the attitudinal assessment, younger children had higher negative feelings about their stutter as compared to adults. Participants also completed a behavioral assessment and the researchers compared results of the behavioral and attitudinal tasks to explore meaningful relationships. There was no significant correlation between the researcher's calculations of severity of stuttering and the participant's self-reported attitudes. This means that, as a general trend across all ages, individuals who have a more severe overt stutter do not necessarily feel more negatively about their stuttering than individuals with a less severe overt stutter. These data are important to note because selfperception of stuttering frequency may be influenced by the negative feelings someone holds about their stuttering. According to Kondrashov and Tetnowski (2019) younger children had more negative feelings about their stuttering/speech production when compared to attitudes of older children and adults.

\subsection{Scale Reliability}

There is little research exploring the relationship between clients' self-perceptions of their stuttering frequency and disfluency count data. O'Brian et al. (2004) compared the reliability of a nine point self-rating scale with a speech-language pathologist's (SLP) calculated percent of stuttered syllables. Their findings showed comparable results between the two methods when a self-rating is completed immediately after speaking, but there was a decrease in accuracy when the rating was delayed post speaking (O’Brian et al., 2004).

Karimi et al. (2014) examined the absolute and relative reliability of rating stuttering on a scale. The results showed that utilizing a scale for rating stuttering frequency is reliable when measuring relative reliability, but less reliable when examining absolute reliability. Thus, when looking at more relative trends, as in this study, scale rating is a reliable method to employ. 


\subsection{Purpose of the Current Study}

The purpose of the current study is to analyze the accuracy of participants' self-ratings of stuttering frequency as compared to clinician calculations of stuttering frequency in order to further examine possible factors that affect the accuracy of a stutterer's self-rating of their overt stuttering. This research contributes additional empirical exploration of stutterer's self-perception of stuttering while also considering variables such as the client's chronological age. Our research question is: Does the age of an individual who stutters impact the accuracy of their self-perception of stuttering frequency, as compared to clinicians' calculation of the stutterer's overt stuttering frequency?

\section{Methods}

\subsection{Participants}

Participants in this study were a 9-year old, a 13-year old, and a 32-year old. The 9-year old participant is a white male who lives in rural Oregon and has participated in the Telepractice Fluency Clinic since 2018. At the time of his initial evaluation, his overt stuttering was noted to be "mild-moderate" as described by the Stuttering Severity Instrument - 4th Edition (SSI-4). His life was noted to be "moderately" impacted by his stuttering, as described by the Overall Assessment of the Speaker's Experience of Stuttering-School-age Version (OASES-S). The 13-year old participant was a white female who lives in a suburban area Oregon and has participated in the Telepractice Fluency Clinic since 2017. At the time of her initial evaluation, her overt stuttering was noted to be "very mild" as described by the SSI-4. Her life was noted to be "mildly" impacted by her stuttering, as described by the Overall Assessment of the Speaker's Experience of Stuttering-Teen Version. The 32-year old participant was a white male who lives in a metropolitan area in Oregon and has participated in the Telepractice Fluency Clinic since 2020. At the time of his initial evaluation, his overt stuttering was noted to be "mild" as described by the SSI-4. His life was noted to be "moderate-severely" impacted by his stuttering, as described by the Overall Assessment of the Speaker's Experience of Stuttering-Adult Version. All participants were seen in conjunction with the Telepractice Fluency Clinic at Portland State University. In 
this clinic, graduate students studying speech-language pathology work under the supervision of a licensed and certified speech-language pathologist with clients who stutter once a week for nine weeks through telepractice. For the purposes of the current study, data was collected and analyzed across the three initial telepractice treatment sessions of the Winter 2021 term for each of the three participants.

\subsection{Experimental Design}

The current study sought to compare clients' self-perception of stuttering severity with clinician collected data to determine how accurate clients who stutter are in self-identifying their own stuttering frequency. Thus, both client self-perception and clinician collected data were utilized.

\subsubsection{Self-Perception Data}

At the end of each telepractice therapy session for three weeks, clients self-reported their perceptions of their stuttering frequency, by responding to the question, "On a scale of 1-5 with one being never and five being always, how would you rate your stuttering frequency during today's session?" Response options were provided on a Likert scale from 1-5. On this scale, $1=$ never, $2=$ rarely, $3=$ sometimes, $4=$ often, and $5=$ always. Participants were asked to rate their overt stuttering self-perception from that day's clinical session alone.

\subsubsection{Clinician Data}

Each of the telepractice therapy sessions were recorded and reviewed asynchronously after the session to code a 300-word sample and calculate the percentage of words stuttered for each client. Disfluency count coding was completed by determining whether a word was fluent or disfluent. If the word was disfluent, it was further coded as a stuttering-like disfluency or a typical disfluency. Stuttering-like disfluencies include audible sound prolongations (ASPs), inaudible sound prolongations (ISPs), single sound repetitions (SSRs), and whole word repetitions (WWRs) as the stuttering behaviors. Typical disfluencies include phrase repetitions (PRs), interjections (INTs), and revisions (REVs). Typical disfluencies are not included in the calculation for percent of stuttered words. If the word was determined to be fluent, it was represented in coding as a dash (non-code). 


\subsection{Interrater Reliability}

After all nine sessions were coded by the author, inter-rater reliability was calculated for $33 \%$ of the sample ( 3 of 9 sessions). A second coder was trained on disfluency count coding under the same system prior to receiving the video recordings of the telepractice therapy sessions for coding. The author and the second coder independently calculated disfluency frequency and types for 300-word samples and then compared their results.

Discrepancies in coding were noted in the coding of inaudible sound prolongations vs interjections in the same disfluent moments. This difference in coding led to a discrepancy in the calculated percent of words stuttered since interjections are considered a "typical" disfluency, and inaudible sound prolongations are a stuttered disfluency. There was also one instance in which the coders identified different types of stuttering-like disfluencies (i.e., ASP and SSR) during a single word production. Discrepancies in coding were discussed by analyzing each portion of a session used for coding word-by-word. After reviewing each session in-depth, final coding frequencies and types were agreed upon by both coders, resulting in 100\% agreement of coding for each session.

\subsection{Analyses}

Client's self-ratings were compared to clinician calculated percentage of words stuttered. The SSI-4 (Riley, 2009) frequency scale for speaking tasks was used as a guideline for matching client's self-ratings with clinician calculated percentages of words stuttered. Each of the numbers on the 5-point Likert scale correlated with a range of percentage of words stuttered. The client's self-ratings were analyzed for accuracy in reference to these predetermined ranges. Table 1 displays the 5-point Likert scale and associated percentage of words stuttered as described in the SSI-4 frequency section 
Table 1: 5-point Likert Scale Terms and \% Words Stuttered Equivalence from the SSI-4

\begin{tabular}{|l|l|l|}
\hline Self-Rating & Likert Terms & Percent of Words Stuttered \\
\hline 1 & Never & $0-4 \%$ \\
\hline 2 & Rarely & $5-9 \%$ \\
\hline 3 & Sometimes & $10-14 \%$ \\
\hline 4 & Often & $15-19 \%$ \\
\hline 5 & Always & $20+\%$ \\
\hline
\end{tabular}

\section{Results}

\subsection{Child Self-Rating and Clinical Data Comparison}

The child client self-rated their stuttering at a " 2 - rarely" at the end of the first two of the three telepractice therapy sessions, and a " 4 - often" at the end of the third telepractice session. The rating "2" correlates to the range of $5-9 \%$ of stuttered words, and the rating " 4 " correlates to the range of $15-19 \%$ of stuttered words. Table 2 describes the clinically calculated percent of words stuttered for each of the three sessions:

Table 2: Frequency of Disfluency Types and \% Words Stuttered for Child Participant

\begin{tabular}{|l|l|l|l|}
\hline Stuttering Type & Session 1 & Session 2 & Session 3 \\
\hline WWRs & 7 & 3 & 2 \\
\hline SSRs & 2 & 4 & 5 \\
\hline ASPs & 1 & 1 & 0 \\
\hline ISPs & 14 & 4 & 4 \\
\hline$\% W S / 300$ & $8 \%$ & $4 \%$ & $3.7 \%$ \\
\hline
\end{tabular}

When the client's self-rating was compared to the clinician calculated data, it was noted that his self-rating was consistent with the clinician's data during the first session, but inconsistent during the second and third sessions. In the first session, the client produced $8 \%$ words stuttered, which was approximately two times the percent of words stuttered during the second (4\%) and third (3.7\%) sessions. 
Despite the decrease in calculated percent of words stuttered, the client increased their self-rating of stuttering severity for the last session. In sum, the client accurately self-rated their stuttering frequency, as compared to the clinician calculated data, in only one of three sessions.

\subsection{Teen Self-Rating and Clinical Data Comparison}

The teen client self-rated their stuttering at a " 2 - rarely" at the end of each of the three telepractice therapy sessions. Table 3 describes the clinically calculated percent of words stuttered for each of the three sessions:

Table 3: Frequency of Disfluency Types and \% Words Stuttered for Teen Participant

\begin{tabular}{|l|l|l|l|}
\hline Stuttering Type & Session 1 & Session 2 & Session 3 \\
\hline WWRs & 0 & 0 & 0 \\
\hline SSRs & 1 & 1 & 0 \\
\hline ASPs & 3 & 0 & 0 \\
\hline ISPs & 3 & 2 & 3 \\
\hline$\% \mathrm{WS} / 300$ & $2.3 \%$ & $1 \%$ & $1 \%$ \\
\hline
\end{tabular}

When the client's self-rating was compared to the clinician calculated data, it was noted that the client consistently overestimated the frequency of their stuttering throughout all three sessions. The client consistently rated their stuttering frequency a " 2 " which correlates to the range of 5-9\% of stuttered words. The highest percent documented in the clinician calculations of stuttering frequency was $2.3 \%$ which is well below the lower limit of the range for the rating of "2" which corresponds to 5-9\% stuttered words.

\subsection{Adult Self-Rating and Clinical Data Comparison}

The adult client self-rated their stuttering at a "3 - sometimes" at the end of each of the three telepractice therapy sessions. Table 4 describes the clinically calculated percent of words stuttered for each of the three sessions: 
Table 4: Frequency of Disfluency Types and \% Words Stuttered for Adult Participant

\begin{tabular}{|l|l|l|l|}
\hline Stuttering Type & Session 1 & Session 2 & Session 3 \\
\hline WWRs & 0 & 0 & 0 \\
\hline SSRs & 1 & 1 & 1 \\
\hline ASPs & 1 & 3 & 0 \\
\hline ISPs & 5 & 5 & 13 \\
\hline$\% \mathrm{WS} / 300$ & $2.3 \%$ & $3 \%$ & $4.7 \%$ \\
\hline
\end{tabular}

When the client's self-rating was compared to the clinician calculated data, it was noted that there

was an increase in percent of words stuttered over the span of the three sessions. The calculated percent of words stuttered in the third session was about two times the percent of words stuttered in the first session. Despite the variability in the clinician calculations from each session, the client maintained a consistent self-rating across sessions.

This client consistently rated their stuttering frequency a " 3 " which correlates to the range of $10-14 \%$ of stuttered words. The highest percentage documented in the clinician calculations of stuttering frequency was $4.7 \%$, which is well below the lower limit of the range for the rating of " 3 ." The adult client consistently overestimated the frequency of their stuttering throughout all three sessions.

\subsection{Overarching Considerations}

All three participants mostly maintained a consistent self-rating of stuttering frequency across their telepractice speech therapy sessions. The child client was the one exception to this consistent rating, as he provided two different ratings across the three sessions. Clients maintained their self-ratings across sessions despite the noted differences in clinician calculations for those sessions. For example, each client had one session that was calculated to have twice as many words stuttered as one of their other three sessions, yet their self-ratings from those sessions held consistent.

Additionally, participants presented with variability in accuracy of their self-rating. Both the teen and adult clients consistently overestimated the frequency of their overt stuttering. The child client was 
accurate with their self-ratings of stuttering frequency for one of the three therapy sessions and was closer to clinician data across all sessions when compared to the teen and adult clients.

Taken together, there is a pattern of maintenance of self-perception despite varying calculated frequencies. There also appears to be a decline in accuracy of self-perception with the increase of age across the clients. Overall, the child client was the most accurate in their self-ratings for stuttering frequency and the adult client was the least accurate in their self-perception of stuttering frequency.

\section{Discussion}

To review, our research question was: Does the age of an individual who stutters impact the accuracy of their self-perception of stuttering frequency, as compared to clinicians' calculation of the stutterer's overt stuttering frequency? The following section will discuss the results of the current study with regard to accuracy of self-perception and the impact of age on a client's self-reported stuttering frequency.

\subsection{Accuracy of Self-reported Stuttering Frequency}

In the current study, two out of three participants showed a tendency to overestimate their stuttering frequency. This inaccuracy was specifically seen in the teen and adult clients where perceptions of their percentage of words stuttered were largely overestimated. This result is inconsistent with O'Brian, et al. (2004) who reported that self-reporting scales used immediately after speaking by stutterers showed comparable results to clinician calculations of stuttering frequency.

One possible explanation for the differences in these results is that the research completed by O’Brian et al. (2004) utilized speech samples from outside of a clinical environment. The environment could lead to variability in where an individual's focus lies. Data collected in a clinical setting, as the data collected in this research was, could be clouded by the client's focus being on other aspects of their stutter and/or stuttering treatment rather than solely frequency. Another factor that could have impacted the findings was the method in which the clinicians calculated the percentage of stuttering. O'Brian et al. (2004) calculated the percent of syllables stuttered for the entire speech sample that the client was 
self-rating. In this research, we only calculated a percent of words stuttered for a 300 -word sample, not the entire session length. Conducting a disfluency count on the whole session, as O'Brian et al (2004) did, may have revealed a more accurate clinician calculation, which in turn, may have led to increased congruence between clients and clinicians.

Another factor that could have impacted the accuracy of self-reported stuttering frequency is the clients' experience(s) of covert stuttering (Douglass et al., 2018). In the current project, only overt stuttering behaviors were coded. Thus, it is possible that the clients experienced additional stuttering moments that were covert in nature which could not be captured by the clinician's data collection. In that case, if the clients were considering both overt and covert stuttering moments, they may have been providing a more accurate description of their stuttering frequency than the clinician's calculated data suggests. Future research should further explore the connection between overt stuttering, covert stuttering, and self-ratings of stuttering frequency.

\subsection{Age and Self-Reported Stuttering Frequency}

To date, no research has comprehensively compared the accuracy of self-perception of stuttering frequency to clinician's calculated percentage of words stuttered across age ranges. Preliminary results of the current study revealed that the child was the most accurate in providing a self-rating of stuttering frequency that aligned with the clinician's calculated stuttering frequency. Accuracy in self-perception of stuttering frequency appeared to decrease with the increase of chronological age, with the adult client being the least accurate in their self-perception of stuttering frequency. As previously noted, covert behaviors may have impacted the clients' self-ratings of their stuttering and, as Douglass et al. (2018) noted, these covert behaviors may increase with age.

\subsection{Stuttering Severity and Self-Reported Stuttering Frequency}

Each client entered with a different level of measured stuttering severity. The child client's overt stuttering was noted to be "mild-moderate" as described by the SSI-4, and their life was noted to be "moderately" impacted by their stuttering, as described by the Overall Assessment of the Speaker's Experience of Stuttering-Child Version. This shows that their overt stuttering level generally aligns with 
their covert stuttering level, and how their stutter impacts their life. This could be a contributing factor in the accuracy seen in this client's self-ratings of stuttering frequency.

The adult client's overt stuttering was "mild" as described by the SSI-4, and their life was noted to be "moderate-severely" impacted by his stuttering, as described by the Overall Assessment of the Speaker's Experience of Stuttering-Adult Version. This shows that the adult client experienced more covert stuttering behaviors than overt stuttering behaviors. This could be a contributing factor in the consistent overestimation seen in their self-ratings of stuttering frequency.

\subsection{Clinical Implications}

The current study provides information for clinicians who are working with clients who stutter. The data show that teenage and adult clients may be less accurate in their self-perception of the frequency of their stutter when compared to a child client. Knowledge of age as a factor in accurate self-ratings of stuttering frequency may play an important role in the communication between client and clinician. If clients' perceptions of their stuttering frequency are consistently overestimated, but their clinicians' notes or reports document a more mild stuttering frequency, clinicians run the risk of invalidating the clients' experience. For example, a client who self-reports that they are stuttering often but whose clinician reports that their stuttering is mild, may feel that the clinician is ignoring the impact of stuttering which may lead to an inconfidence in the therapeutic-alliance. Zebrowski (2002) discussed the importance of building a clinical relationship, specifically with teen clients who stutter, stating that "forming a strong relationship provides the foundation for change" (Zebrowski, 2002, p. 92). Thus, if there is a level of distrust from the client in the clinician, there will not be a strong foundation from which to make positive clinical progress.

Although these results are preliminary in nature, clinicians working with clients who stutter should consider that the SSI-4 only explores the overt stuttering, but is frequently utilized by clinicians as the only tool to determine stuttering severity. Clinicians should consider using both an overt stuttering metric and a covert stuttering metric (such as the Overall Assessment of the Speaker's Experience of Stuttering, Yaruss \& Quesal, 2010) when diagnosing and assigning a severity of stuttering so as to better 
capture a full image of the client's experience. With a better understanding of a client's full stuttering experience, clients and clinicians can move forward with more trust, and understanding in order to create a more positive clinical experience.

\subsection{Limitations and Future Directions}

This research is preliminary in nature and there are several limitations that should be taken into consideration when analyzing and generalizing the results. One limitation is the small sample size $(\mathrm{N}=3)$ used in this study. With such a small sample size, these findings cannot be generalized to a larger group of each chronological age observed. Future research should utilize a larger sample size to gain a better understanding of the impact of age, lived experiences, and covert behaviors on self-rating of stuttering frequency.

An additional limitation is that this research was conducted over the span of three telepractice clinical sessions due to researcher time-constraints. Three weeks equates to only one third of the clients' telepractice sessions for the Winter 2021 term. Future research should pursue a longitudinal study, across nine weeks or longer. This would allow for more nuanced examination of the age, covert/overt stuttering, and self-perception factors for each client and across the lifespan.

A third limitation of the method used in this study is the use of the SSI-4 as a rating scale for comparison with the client's self-rating of stuttering frequency. The scale used in the SSI-4 has a rating of " 0 " that correlates to more than $0 \%$ of stuttered words (in this research the range is $0-4 \%$ ). The self-rating of " 0 " is described to the clients as a rating of "never", but is not actually equivalent to $0 \%$ words stuttered, possibly misleading clients and contributing to their overestimation of their stuttering frequency. The clients may have reached a higher level of accuracy if the scale utilized in this research had a rating of "never" that was equivalent to $0 \%$, and the rating of "rarely" began at $1 \%$ of words stuttered.

A final limitation is the lack of tracking/coding of covert stuttering in addition to the coding of overt stuttering behaviors. As noted by Douglass et al. (2018), overt stuttering does not encompass the totality of the experience of stuttering. Covert stuttering enacts all of the same psychological processes as overt stuttering (Douglass et al., 2018), and therefore would likely be a factor impacting an individual's 
self-perception of their stuttering frequency. Given the limitations discussed above, the results of this study are preliminary in nature and should be interpreted with caution.

\section{Conclusion}

The current study sought to explore the relationship of clients' self-ratings of stuttering when compared to clinician calculated percentage of words stuttered. All three clients in the current study overestimated the frequency of their stuttering when compared to clinician coding. Additionally, there appears to be an inverse relationship between chronological age and accuracy of clients' self-perceptions of stuttering frequency was noted, with the younger client reporting more accurate self-ratings of stuttering than the teen or adult. Future research should further explore the relationships between age, covert stuttering, and self-perception of stuttering frequency. 


\section{References}

Alameer, M., Meteyard, L., \& Ward, D. (2017). Stuttering generalization self-measure:

Preliminary development of a self-measuring tool. Journal of Fluency Disorders, 53, 41-51. doi:10.1016/j.jfludis.2017.04.001

ASHA. (2021). Stuttering. ASHA. https://www.asha.org/

Douglass, J. E., Schwab, M., \& Alvarado, J. (2018). Covert stuttering: Investigation of the paradigm shift from COVERTLY stuttering to OVERTLY STUTTERING. American Journal of Speech-Language Pathology, 27(3S), 1235-1243. doi:10.1044/2018_ajslp-odc11-17-0190

Harvard Health Publishing. (2018). Stuttering. https://www.health.harvard.edu/a_to_z/stuttering-a-to-z.

Karimi, H., O’Brian, S., Onslow, M., \& Jones, M. (2014). Absolute and Relative Reliability of Percentage of Syllables Stuttered and Severity Rating Scales. Journal of Speech, Language, and Hearing Research, 57(4), 1284-1295. doi: 10.1044/2014_jslhr-s-13-0106

Kondrashov, S. M., \& Tetnowski, J. A. (2019). Perceptions of Stuttering of Different Age Groups. Perspectives of the ASHA Special Interest Groups, 4(6), 1311-1315. doi: 10.1044/2019_pers-sig4-2018-0007

O’Brian, S., Packman, A., Onslow, M., \& O’Brian, N. (2004). Measurement of Stuttering in Adults: Comparison of Stuttering-Rate and Severity-Scaling Methods. Journal of Speech, Language, and Hearing Research, 47(5), 1081-1087. doi: 10.1044/1092-4388(2004/080)

O’Brian, S., Packman, A., \& Onslow, M. (2004). Self-Rating of Stuttering Severity as a Clinical Tool. American Journal of Speech-Language Pathology, 13(3), 219-226. doi:

$10.1044 / 1058-0360(2004 / 023)$

Riley, G. D. (2009). Stuttering Severity Instrument-Fourth Edition (SSI-4) [Examiner Record Form]. 
Published by: Pro-Ed.

The Stuttering Foundation. (2021). Frequently Asked Questions. https://www.stutteringhelp.org/faq

Tichenor, S. E., \& Yaruss, J. S. (2019). Stuttering as defined by adults who stutter. Journal of Speech, Language, and Hearing Research, 62(12), 4356-4369. doi:10.1044/2019_jslhr-19-00137

Yaruss, J. \& Quesal, R. (2010). The Overall Assessment of the Speaker's Experience of Stuttering. Published by: Stuttering Therapy Resources.

Zebrowski, P. M. (2002). Building clinical relationships with teenagers who stutter. Contemporary Issues in Communication Science and Disorders, 29(Spring), 91-100. doi:10.1044/cicsd_29_s_91 